\title{
The equatorial E-region and its plasma instabilities: a tutorial
}

\author{
D. T. Farley \\ School of Electrical and Computer Engineering, Cornell University, Ithaca, NY, USA
}

Received: 7 October 2008 - Revised: 19 February 2009 - Accepted: 19 February 2009 - Published: 2 April 2009

\begin{abstract}
In this short tutorial we first briefly review the basic physics of the E-region of the equatorial ionosphere, with emphasis on the strong electrojet current system that drives plasma instabilities and generates strong plasma waves that are easily detected by radars and rocket probes. We then discuss the instabilities themselves, both the theory and some examples of the observational data. These instabilities have now been studied for about half a century (!), beginning with the IGY, particularly at the Jicamarca Radio Observatory in Peru. The linear fluid theory of the important processes is now well understood, but there are still questions about some kinetic effects, not to mention the considerable amount of work to be done before we have a full quantitative understanding of the limiting nonlinear processes that determine the details of what we actually observe. As our observational techniques, especially the radar techniques, improve, we find some answers, but also more and more questions. One difficulty with studying natural phenomena, such as these instabilities, is that we cannot perform active cause-and-effect experiments; we are limited to the inputs and responses that nature provides. The one hope here is the steadily growing capability of numerical plasma simulations. If we can accurately simulate the relevant plasma physics, we can control the inputs and measure the responses in great detail. Unfortunately, the problem is inherently three-dimensional, and we still need somewhat more computer power than is currently available, although we have come a long way.
\end{abstract}

Keywords. Ionosphere (Electric fields and currents; Equatorial ionosphere; Ionospheric irregularities)

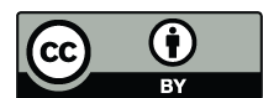

Correspondence to: D. T. Farley (donf@ece.cornell.edu)

\section{Introduction}

Plasma instabilities in the ionospheric E-region are driven by the currents that flow at E-region altitudes and by the electron density gradients there. We now know that these instabilities can be detected at times at any latitude, but they are most common, and strongest, at equatorial latitudes and in the auroral zone, where they have been detected by radars for at least six decades. The instabilities generate plasma waves, with phase fronts highly aligned with the geomagnetic field; any waves that are not so aligned are rapidly destroyed by diffusive damping.

The currents that drive the instabilities flow perpendicular to the geomagnetic field. Such currents are particularly strong in the auroral zone and at the equator. The high latitude currents are often very strong because the electric fields, driven by the solar wind and magnetospheric dynamo, can be very strong: $50-100 \mathrm{mV} /$ meter is not uncommon. At equatorial latitudes, which are our concern here, the currents and electric fields are driven by tidal winds at low and middle latitudes and are weaker, but stronger than at mid latitudes.

The current flow patterns at low latitudes are determined both by the winds and by the conductivities, which are small at night because of the low electron densities, and vary strongly with altitude during the day. The strongest tidal winds in the E-region are driven by the 24-h "tide" associated with solar heating. To lowest order, the winds flow away from the subsolar point towards midnight. In the lower E-region the electrons are strongly magnetized $\left(\Omega_{e} \gg v_{e}\right)$ but the ions are not $\left(\Omega_{i} \ll v_{i}\right)$, where $\Omega$ is the gyro frequency and $v$ is the collision frequency with neutral molecules. So the ions at first try to follow the wind (with velocity $\mathbf{U}$ ), but the electrons try to move in the $-\mathbf{U} \times \mathbf{B}$ direction. This produces a current, but this current must be essentially divergence free, and polarization charges arise to ensure this. The resulting current pattern is complicated by the fact that the conductivity is inhomogeneous and anisotropic.

Published by Copernicus Publications on behalf of the European Geosciences Union. 
The upshot of all this is that the daytime solar quiet $\left(S_{q}\right)$ low latitude E-region currents flow approximately counter clockwise in the Northern Hemisphere and clockwise in the southern, with foci at roughly $\pm 30^{\circ}$ magnetic latitude at the equinoxes. The details of this pattern vary with season and longitude, the latter due to the fact that the magnetic equator deviates substantially from the geographic equator in some locations (especially South America). The equatorial electrojet currents are strong not because the equatorial zonal electric fields are strong (they are usually quite weak, on the order of 0.5 to $1 \mathrm{mV} / \mathrm{m}$ typically), but because the conductivities are unusually high in the narrow latitude region where the geomagnetic field is very nearly horizontal. The $S_{q}$ current system has been studied for decades using ground based magnetometers. During strong magnetic disturbances driven by the magnetosphere and solar wind, the current pattern can become quite distorted, and sometimes it actually reverses (producing a counter electrojet). As we shall learn, such a reversal has interesting consequences for the plasma instabilities.

In the sections to follow we look first at some basic photochemistry and related time constants, and next at the conductivity tensor and what happens near the magnetic equator. Much of this material follows standard treatments in texts such as Rishbeth and Garriott (1969) and Kelley (1989). Then we move on to consider the basic features of the Eregion plasma instabilities and some of the assumptions that are commonly made for the equatorial case. This tutorial is meant to be an introduction to the instabilities rather than a complete review of five decades of work. A very thorough discussion of most of the current state of the art has recently been given by Hysell et al. (2007).

\section{Production and loss of ionization}

The equation of continuity for the electron concentration $N$ is

$\frac{\partial N}{\partial t}=q-\ell(N)-\operatorname{div}(N \mathbf{V})$

where $q$ is the production rate, $\ell(N)$ is the loss rate, and the last term is the transport term ( $\mathbf{V}$ is the velocity). Transport is usually not important during the day in the E-region (but it may matter at night for long lived metallic ion layers). And for the zero order densities the time derivative is usually negligible and the production and loss terms balance each other, producing photochemical equilibrium. The time derivative and transport terms do matter, however, when we consider plasma instabilities.

The production is due to photoionization by solar extreme ultraviolet (EUV) radiation that produces mostly molecular ions, plus some metallic atomic ions associated with meteor debris. The most important loss mechanism in the E-region is dissociative recombination, described by

$X Y^{+}+e \rightarrow X^{*}+Y^{*}$ where the asterisks indicate excited states, and with a rate coefficient $\alpha_{e} \sim 10^{-13} \mathrm{~m}^{3} \mathrm{~s}^{-} 1$, meaning that

$\frac{\partial N}{\partial t}=\alpha_{e}[N]\left[X Y^{+}\right]=\alpha_{e} N^{2}$

so for typical equatorial electron and ion daytime densities of the order of $10^{11} \mathrm{~m}^{-3}$, the decay time $\tau=\left[N^{-1} \partial N / \partial t\right]^{-1}$ would be about $10^{2} \mathrm{~s}$, which is very short compared to a day (but very long compared to the time scales $\lambda / V$ of all but very long plasma waves). The transport term time constant will also be very long for the zero order profile since the ambient electron density gradients are essentially vertical and the velocities are essentially horizontal.

At night, however, the situation is quite different. The molecular ion densities are one or two orders of magnitude smaller, so the dissociative recombination times are correspondingly longer, and sometimes metallic ions may dominate the density profile, in which case we must consider radiative recombination, namely

$X^{+}+e \rightarrow X^{*} \rightarrow X+h v$

with now a much smaller rate coefficient of $\alpha_{e} \sim 10^{-18} \mathrm{~m}^{3} \mathrm{~s}^{-1}$, corresponding to a time constant of many days! So there is plenty of time for suitable winds and electric fields to generate thin layers with relatively high density. (The reason that $\alpha_{e}$ is so much smaller for Eq. (4) than for Eq. (2) is that it is much harder to conserve both energy and momentum for Eq. (4).)

Attachment and detachment of electrons to and from neutral molecules can also destroy or create free electrons, but these processes are not important at the altitudes we are interested in.

\section{Equatorial electrojet conductivities}

The electrical conductivity in the ionosphere is highly anisotropic, which we describe with the tensor

$\boldsymbol{\sigma}=\left[\begin{array}{ccc}\sigma_{p} & 0 & \sigma_{h} \\ 0 & \sigma_{0} & 0 \\ -\sigma_{h} & 0 & \sigma_{p}\end{array}\right]$

where the Cartesian coordinates point eastward, northward (parallel to B), and upward at the equator, and the parallel, Pedersen, and Hall conductivities are described by

$\frac{\sigma_{0}}{N e^{2}}=k_{0 e}+k_{0 i}, \frac{\sigma_{p}}{N e^{2}}=k_{p e}+k_{p i}, \frac{\sigma_{h}}{N e^{2}}=k_{h e}-k_{h i}(6)$ where the mobilities are given by

$m v k_{0}=1, \quad m v k_{p}=\frac{v^{2}}{v^{2}+\Omega^{2}}, \quad m v k_{h}=\frac{v \Omega}{v^{2}+\Omega^{2}}$

In the last line we use the values of mass $m$, collision frequency (with neutrals) $v$, and Larmor frequency $\Omega=|e| B / m$ (taken to be always positive) for the (singly charged) ions or 

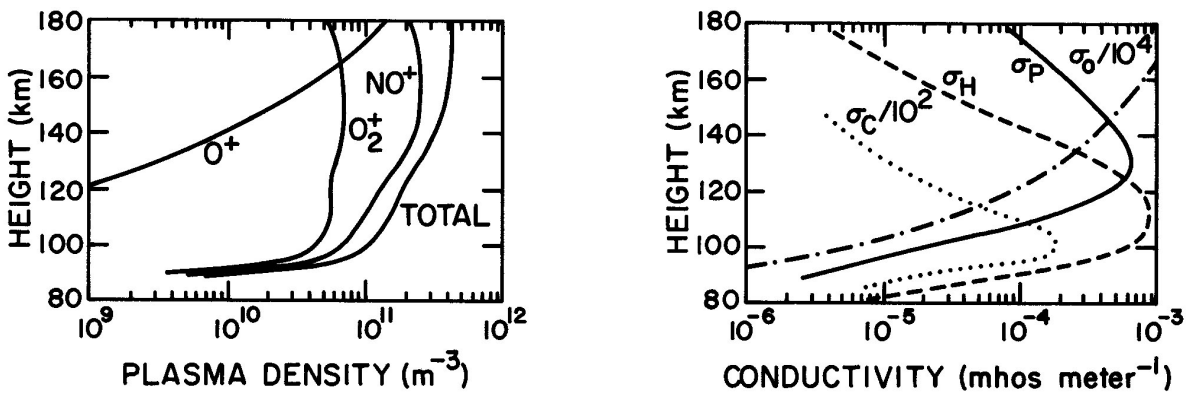

Fig. 1. Vertical profiles of daytime composition and plasma density (left) and conductivities (right) for average solar conditions (from Forbes and Lindzen, 1976.)

electrons, as appropriate. We also note that the electron term dominates the parallel and Hall conductivities, whereas the ions dominate the Pedersen conductivity. For example, for an eastward $\mathbf{E}$, the electrons would $\boldsymbol{E} \times \boldsymbol{B}$ drift upward, corresponding to a downward (negative) vertical current, while the ions would move slowly eastward. Some typical conductivity profiles are shown in Fig. 1.

The daytime low and middle latitude horizontal $S_{q}$ currents are driven fundamentally by the poleward winds described in the Introduction interacting with the vertical component of the geomagnetic field. This vertical component is of course very small at low latitudes, so the wind driven currents flow mainly westward (eastward electron drift) at latitudes above 30 degrees or so. In the nighttime hemisphere the E-region densities are very low and so, to maintain $\nabla \cdot J=0$, a positive (negative) charge builds up at the dawn (dusk) terminator. This charge distribution drives the eastward daytime equatorial return current. The associated eastward electric field, as already mentioned, is comparatively weak, perhaps $0.5 \mathrm{mV} / \mathrm{m}$. Why are the electrojet currents so strong?

This happens because vertical currents are strongly inhibited at the magnetic equator, since no vertical current flows parallel to the magnetic field (and $\sigma_{0} \gg \sigma_{h}, \sigma_{p}$ ). Below $90 \mathrm{~km}$ or so the conductivity decreases rapidly because of the increasing collision frequencies, and above $140 \mathrm{~km}$ ions become magnetized and so the electrons and ions $\boldsymbol{E} \times \boldsymbol{B}$ drift together and the Hall conductivity drops. As a crude approximation, then, we can consider the electrojet layer, centered at about $103-105 \mathrm{~km}$, to be bounded above and below by insulating regions. Polarization charges build up on these boundaries to (almost) prevent vertical current flow in the layer. Then from Eq. (5) we find that

$J_{z}=-\sigma_{h} E_{x}+\sigma_{p} E_{z} \simeq 0 \Longrightarrow E_{z} \simeq \frac{\sigma_{h}}{\sigma_{p}} E_{x}$

The Hall/Pedersen conductivity ratio reaches a maximum value of $15-20$ in the vicinity of $103-105 \mathrm{~km}$, and so the upward polarization field is an order of magnitude larger than the original driving zonal field. This field now drives an east-

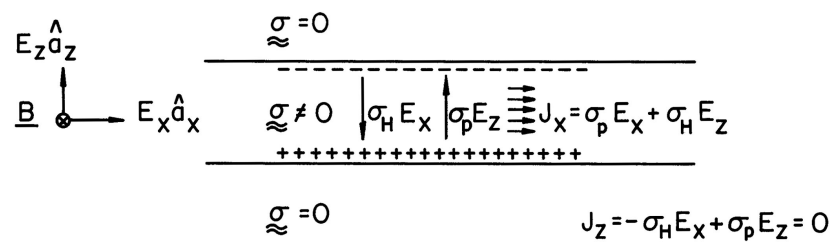

Fig. 2. The slab geometry model of the equatorial electrojet (from Kelley, 1989).

ward Hall current (consisting mainly of a westward electron drift), and so the effective conductivity in terms of the original zonal electric field is increased from $\sigma_{p}$ to what is known as the Cowling conductivity

$\sigma_{\text {Cowling }}=\frac{\sigma_{h}^{2}}{\sigma_{p}}+\sigma_{p}$

The slab model geometry is shown in Fig. 2.

If we are slightly off the magnetic equator, at a dip angle of $I$, a little algebra shows that this last result would be

$\sigma_{x x}=\frac{\sigma_{h}^{2} \cos ^{2} I}{\sigma_{0} \sin ^{2} I+\sigma_{p} \cos ^{2} I}+\sigma_{p}$

which reduces to the Cowling result for $I \ll \sqrt{\sigma_{p} / \sigma_{0}}$, i.e., a few degrees or less. So the equatorial electrojet is confined to a narrow band of latitude only a few hundred kilometers wide.

Farther off the equator the vertical current may not be zero because field aligned currents will flow in response to northsouth asymmetries in the global wind system, in order to nearly equalize the potentials at opposite ends of the magnetic field lines.

Another way of looking at the electrojet polarization process is in terms of the electron drift velocity, which is what really matters for the instability discussion to follow. If we start with a daytime zonal, eastward electric field of $0.5 \mathrm{mV} / \mathrm{m}$, the induced upward polarization field will be roughly $10 \mathrm{mV} / \mathrm{m}$, 
which will produce a westward electron drift velocity $E / B$ of about $400 \mathrm{~m} / \mathrm{s}$ ( $B \simeq 0.25$ gauss at the equator), which will excite unstable, westward traveling plasma waves, as we shall see. The typical noontime electrojet current density reaches maximum values of the order of $10^{-5} \mathrm{amps} \mathrm{m}^{-2}$ and causes a typical midday increase in the equatorial (horizontal) geomagnetic field of the order of $100 \mathrm{nT}$.

This slab model of the equatorial electrojet was essentially verified by the 1994 in-situ rocket measurements in Brazil (the Guará campaign) described by Pfaff et al. (1997). The vertical polarization field is difficult to measure since the rocket has to be oriented parallel to the magnetic field, but this was achieved in 1994, and the authors found a maximum value of the daytime vertical electric field near the altitude of $105 \mathrm{~km}$ to be about $9 \mathrm{mV} / \mathrm{m}$, corresponding to a westward electron drift of about $360 \mathrm{~m} / \mathrm{s}$. Some details of the data did not fully agree with the simple model, but the key feature of the large induced vertical polarization field driving a large Hall current was fully confirmed.

At night this whole scenario reverses sign and the currents are westward. The nighttime currents are much weaker, however, because the electron densities are so much smaller, and so they cannot be studied effectively by magnetometer networks on the ground. The electric fields and the electron drift velocities, however, are comparable to the daytime values, and so again can easily drive plasma instabilities.

The other important parameter for the instabilities is the electron density profile, which is smooth with an upward gradient until about $107 \mathrm{~km}$ or so during the day, but becomes very jagged with sharper gradients and lower densities at night.

\section{Electrojet plasma instability theory}

\subsection{Basic ideas}

We begin with a quick summary of the well known linear fluid theory of the equatorial electrojet instabilities and then review the perhaps not quite so familiar assumptions that are usually made for the equatorial electrojet case (only; some of these assumptions are not valid at high latitudes). The plasma waves are electrostatic, longitudinal waves (wave vectors parallel to the wave electric field and velocity perturbations). The equations we need are the continuity equations already given in Eq. (1) for the ions and electrons, the equations of motion or momentum (for no neutral wind)

$m N \frac{d \boldsymbol{V}}{d t}=q N(-\nabla \Phi+\boldsymbol{V} \times \boldsymbol{B})-\nabla P-m v N \boldsymbol{V}$

for the ions and electrons ( $\Phi$ is the electrostatic potential, $P$ is the particle pressure, and $\boldsymbol{B}$ is the (constant) geomagnetic field in the above), and Poisson's equation

$\nabla^{2} \Phi=-\left(e / \epsilon_{0}\right)\left(N_{i}-N_{e}\right)$
For scale lengths much larger than the Debye length $\left(\lambda_{D} \sim 3 \mathrm{~mm}\right.$ in the electrojet region), Poisson's equation can be replaced with $N_{e} \approx N_{i}=N$. We also, for now, set $P=N K T$, the isothermal case, with $K$ being Boltzmann's constant.

The next step is to linearize all the equations, with the perturbations being sinusoidal traveling waves, i.e.,

$N \rightarrow N_{0}+n \exp [i(\mathbf{k} \cdot \mathbf{r}-\omega t)]$

with similar expansions for $\Phi$ and $V$. Such "local" plane wave solutions are valid so long as the wavelengths of interest are small compared to the dimensions of the electrojet. We now eliminate the zero order terms, put the equations for the first order perturbations in matrix form, noting that $\partial / \partial t \rightarrow-i \omega$ and $\nabla \rightarrow i \mathbf{k}$ for the perturbations, i.e.

$$
\left[\begin{array}{ccc}
a_{11} & \cdots & a_{n 1} \\
\vdots & \ddots & \vdots \\
a_{1 n} & \cdots & a_{n n}
\end{array}\right]\left[\begin{array}{c}
b_{1} \\
\vdots \\
b_{n}
\end{array}\right]=0
$$

where the column vector consists of the unknown perturbations, and then we set the determinant of the coefficient matrix equal to zero to find the dispersion equation relating $\omega$ to the wave vector $\boldsymbol{k}$. The last step is to set the (complex) $\omega=\omega_{k}+i \gamma_{k}$ and further assume that the magnitude of the growth (or damping) rate $\left|\gamma_{k}\right| \ll\left|\omega_{k}\right|$.

All this leads finally to the "standard" local linear fluid theory (e.g., Fejer et al., 1975, and several earlier references) for the equatorial electrojet plasma waves in the frame of the ions (essentially the frame of the neutral wind), namely

$\omega_{k}=\frac{\boldsymbol{k} \cdot \boldsymbol{V}_{d}}{1+\psi}$

for the real part of the angular oscillation frequency and

$\gamma_{k}=\frac{\psi}{(1+\psi) \nu_{i}}(\underbrace{\omega_{k}^{2}}_{1}-\underbrace{k^{2} C_{s}^{2}}_{2})+\underbrace{\frac{v_{i}}{\Omega_{i}} \frac{\omega_{k} k_{\mathrm{west}}}{L k^{2}}}_{3}-\underbrace{2 \alpha N}_{4}$

for the growth rate $\left(\mathrm{s}^{-1}\right)$, where

$\psi(\theta)=\psi_{0}\left(\frac{k_{\perp}^{2}}{k^{2}}+\frac{\Omega_{e}^{2} k_{\|}^{2}}{v_{e}^{2} k^{2}}\right) \approx \frac{v_{e} v_{i}}{\Omega_{e} \Omega_{i}}\left(1+\frac{\Omega_{e}^{2}}{v_{e}^{2}} \theta^{2}\right)$

and $L=n_{0}\left(d n_{0} / d h\right)^{-1}$ is the vertical gradient length (which is assumed for a local theory to be large compared to wavelengths of interest), $C_{s}$ is the isothermal (usually) ionacoustic velocity, $\mathbf{V}_{d}$ is the electron drift velocity relative to the ions, $\alpha$ is the recombination rate, $\mathbf{k}=\mathbf{k}_{\text {plasma }}$ is the wave vector (with a westward component $k_{\text {west }}$ ), and the aspect angle $\theta$ is the complement of the angle between $\mathbf{k}$ and $\mathbf{B}$, and so $\theta \rightarrow 0 \Rightarrow \mathbf{k} \perp \mathbf{B}$. The aspect angle effects are all contained in the last term in Eq. (17). For radar backscatter $\mathbf{k}_{\text {plasma }}=2 \mathbf{k}_{\text {radar }}$.

The growth rate Eq. (16) includes four parts: (1) the destabilizing effect of ion inertia, (2) diffusive stabilization, 


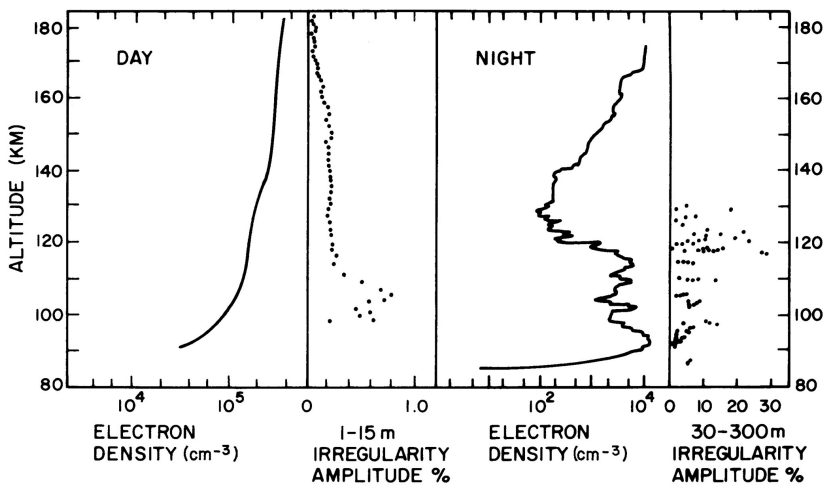

Fig. 3. Electron density profiles and irregularity amplitudes over Thumba, India around noon and midnight. Note the effects of the density gradients (rom Prakash et al., 1972).

(3) the destabilizing or stabilizing effect of the electron density gradient, and (4) stabilization by recombination, which is negligible except for very long wavelengths and the associated long time scales. Recombination is unimportant for the wavelengths of a few meters or less associated with radar echoes but usually limits very long, horizontally propagating waves to lengths no greater than a kilometer or two during the day. The waves can be significantly longer at night, when the recombination is substantially reduced, and then nonlocal effects (which we neglect here) may become important.

The crucial density gradient term is always positive (upward) during the day to altitudes of at least $105-107 \mathrm{~km}$ due to the photochemical equilibrium; at higher altitudes the daytime gradient becomes very small or even slightly negative (Pfaff et al., 1987a), as we shall see later. In the evening, as the layer decays, however, the positive gradient will extend higher, and at night, once the electron densities have dropped by one or two orders of magnitude, the density profile is controlled by dynamics, not photochemistry, and becomes very jagged, with upward and downward gradients (positive and negative values of $L$ ) throughout the region.

So the gradient term (3) is always destabilizing throughout most of the electrojet region during the day for the normal eastward current flow (westward electron drift and westward traveling waves; i.e., positive values of $L$ for a positive $k_{\text {west }}$ ). For weak electric fields (drift velocities $V_{d}$ much smaller than $C_{s}$ but not zero, so that $\omega_{k}$ has the proper sign), we can ignore term (1) as well as (4) in Eq. (16). Then, for long enough wavelengths (but not so long that neglected nonlocal effects become important), term (3) will always be larger than term (2) and the waves will grow. If the normal dynamo electric field should reverse during the day, as it sometimes does (counter electrojet), the unstable waves completely disappear unless the reversed drift is very large (which is rare). Excellent examples of both of these counter electrojet cases are given in Woodman and Chau (2002).
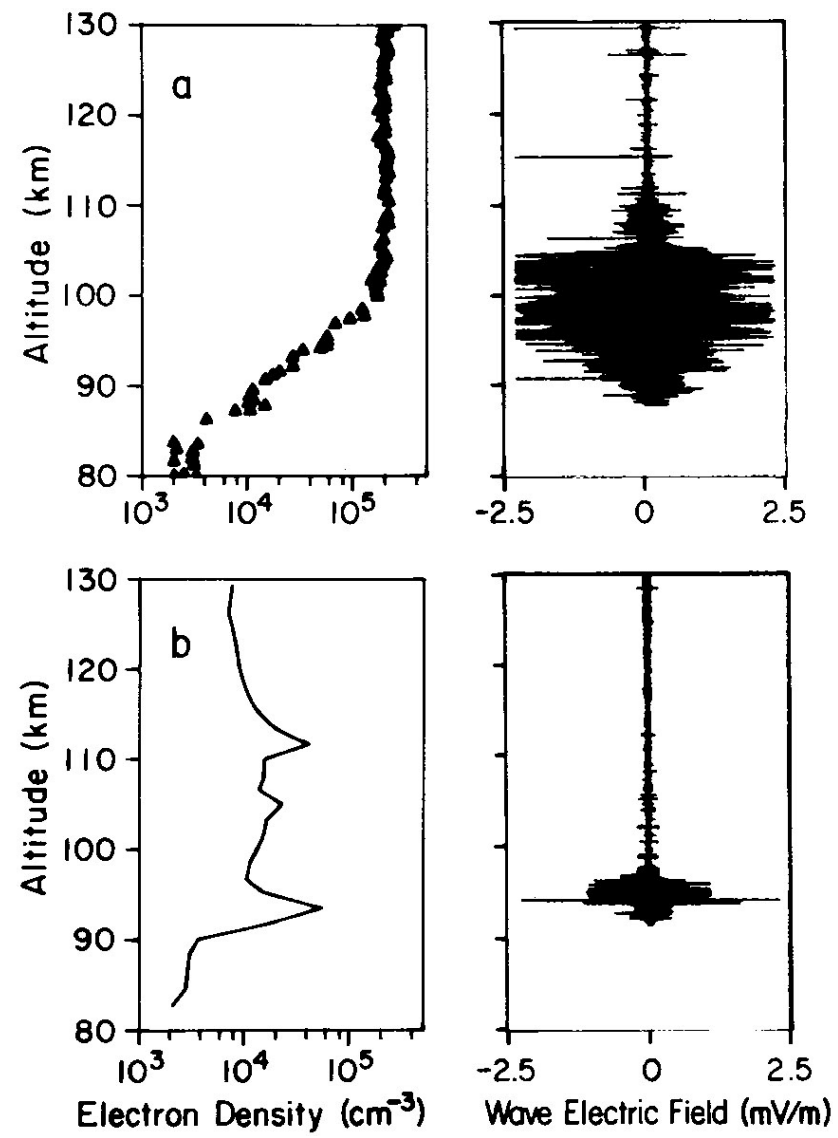

Fig. 4. Daytime (Peru, 1975; panel a) and nighttime (Kwajalein, 1978; panel b) rocket observations of density profiles and wave electric fields (from Pfaff et al., 1982).

At night, except near the time of the daily wind-driven dynamo electric field reversals, long wavelengths should always be unstable; there will be regions of instability for either eastward or westward $V_{d}$.

Some early examples of daytime and nighttime rocket observations illustrate these points. Figure 3 shows daytime and nighttime data over Thumba, India. During the day the irregularities disappear (except for residual noise) above $110 \mathrm{~km}$ or so, where the density profile flattens; but at night, when the profile is jagged, the irregularities cover a much wider range of altitudes. Figure 4 shows (a) daytime data from Peru in 1975 and (b) nighttime data from Kwajalein in 1978. Again, during the day the irregularities are seen where the density gradient is upward, while at night the reverse is true. And note that the Indian data show longer wavelengths and higher altitudes for the irregularities at night. The Indian and Peruvian observations are very close to the magnetic equator, whereas the dip angle is about $9^{\circ}$ at Kwajalein. The instability is not quite as strong there as a result, but the same basic ideas apply. 


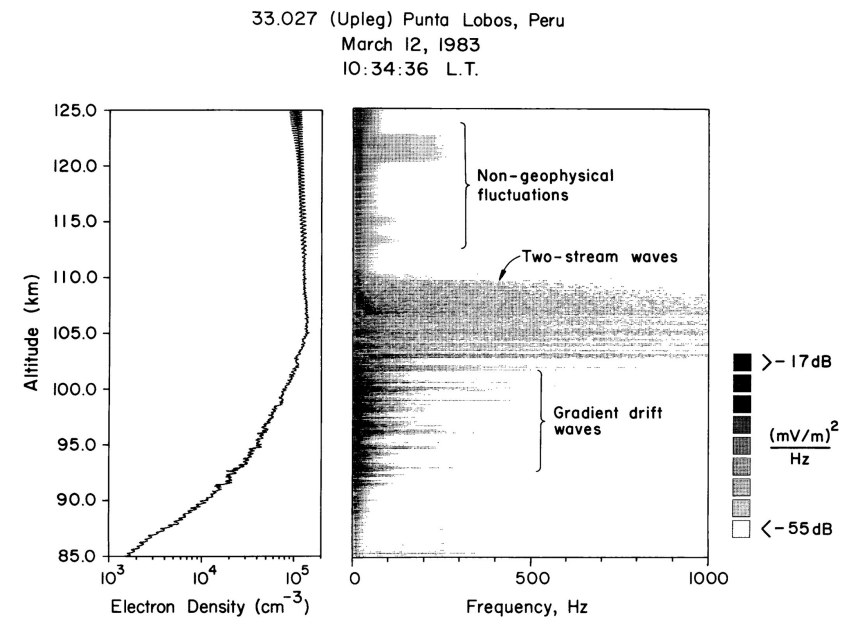

Fig. 5. Rocket observations of density gradients and wave activity. The waves change character where the profile flattens out (from Pfaff et al., 1987a).

\section{Horizontal Electric Fields (Upleg) \\ 33.027 - Punta Lobos, Peru}
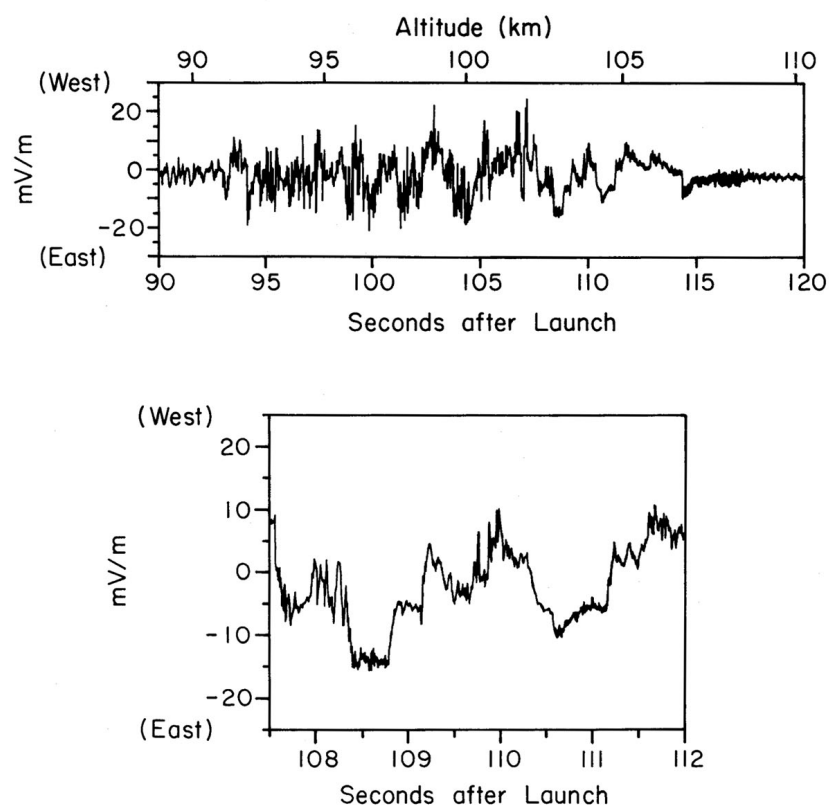

Fig. 6. Daytime (10:34 LT on 12 March 1983) rocket observations of horizontal electric fields over Peru due to large scale waves. Note that the magnitudes are comparable to typical strong vertical polarization fields and are much larger than the eastward $S_{q}$ field (from Pfaff et al., 1987a).

Some additional interesting rocket data are shown in Figs. 5, 6 and 7. The first figure shows a daytime density profile over Peru that flattens out at about 105 or $106 \mathrm{~km}$.

\author{
Vertical Drift Velocities \\ 33.027 - Punta Lobos, Peru
}
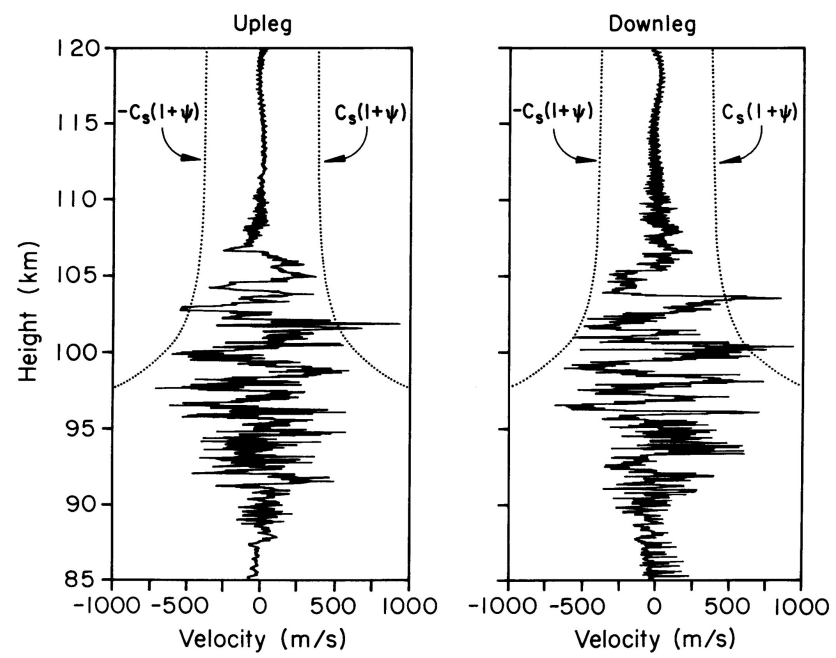

Fig. 7. Comparing the rocket data of the previous figure to the ionacoustic threshold (from Pfaff et al., 1987b).

Below that height there are strong long wavelength waves. Above that height there is still some strong wave activity for a couple of kilometers, but these waves were short and were traveling mostly horizontally. In Fig. 6 we see that the horizontal wave electric fields can exceed $10 \mathrm{mV} / \mathrm{m}$. These observations, taken during the Condor campaign of 1983, are still the only in-situ observations taken during "two-stream" (see next paragraph) conditions. The very strong zonal perturbation fields, comparable to the vertical polarization field and at least an order of magnitude larger than the zero order mean zonal field, show that the electrojet is not at all a nice laminar electron flow. Rather it is usually a highly turbulent flow whose mean velocity is (nearly but not quite) horizontal. Figure 7 shows the same wave data, but plotted differently to show roughly how it compares to the "two-stream" threshold velocity.

In the literature authors refer to (a) the "ion-acoustic" or "FB" or "two-stream" instability when term (1) in Eq. (16) exceeds term (2) and both are much greater than term (3), and to (b) the "gradient-drift" instability when (1) is small and (3) is positive and exceeds (2). In fact, of course, there is really only one dispersion relation and one instability, but with two driving terms and two limiting cases, which depend on the plasma wavelength in quite different ways; the gradientdrift term dominates for long wavelengths. Case (a) produces radar echoes known as "type 1" for historical reasons. These echoes are strong and have a Doppler spectrum that is sharply peaked at a phase velocity comparable to or somewhat greater than the ion-acoustic velocity $C_{s}$, usually at all radar zenith angles, which is perhaps surprising. Case (b) 


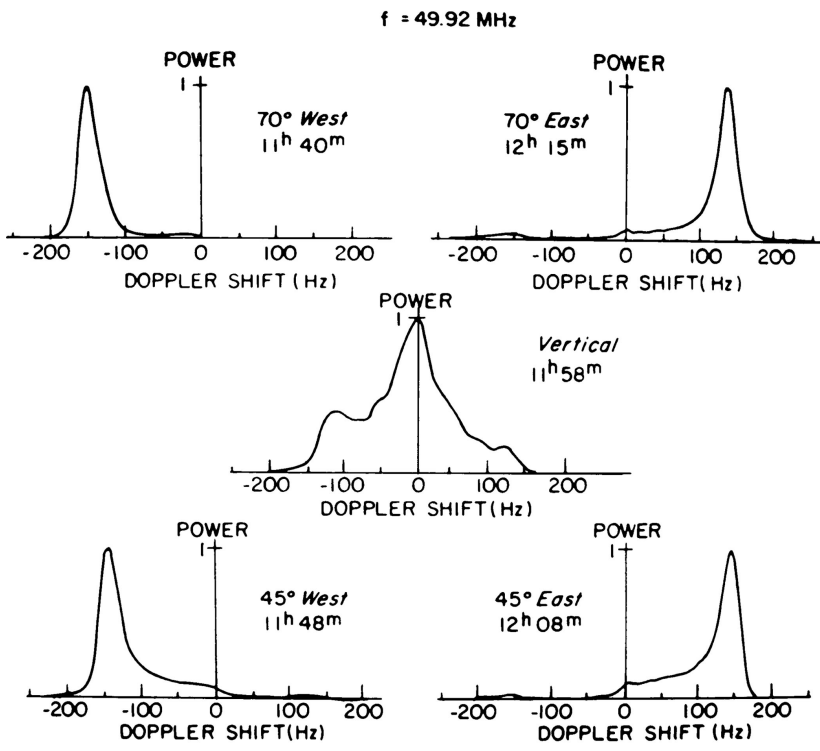

Fig. 8. Early Doppler spectra observations at Jicamarca at various zenith angles during a strong electrojet showing both type 1 and type 2 echoes. Note that the position of the echo peaks for the oblique echoes is nearly independent of zenith angle (from Cohen and Bowles, 1967).

corresponds to radar echoes known as "type 2". These are weaker and have a broader Doppler spectrum with a peak in the vicinity of $\omega=\mathbf{k}_{\text {plasma }} \cdot \mathbf{V}_{d}$. Figures 8 and 9 show examples from the Jicamarca Observatory in Peru in the 1960s of these two kinds of radar echoes.

Figure 8 shows type 1 echoes from overhead and from two substantially different oblique angles (both east and west), and both with almost the same Doppler shift, whereas Fig. 9 shows broader spectra with peaks whose position varies with the sine of the zenith angle, in general agreement with Eq. (15). The type 1 echoes, on the other hand, have their peak Doppler shift close to the value at the threshold of growth determined by the first two terms in Eq. (16). This last equation does not include kinetic effects, which can be significant. The effect of these is that the ion-acoustic velocity $C_{s}$ increases somewhat with decreasing wavelength. This dependence was nicely illustrated in an early paper (Balsley and Farley, 1971) that compared simultaneous radar observations at radar frequencies of 16,50 , and $146 \mathrm{MHz}$. A sample of that data is shown in Fig. 10. Note the systematic changes in the type 1 peaks. No type 2 echoes were observed at $146 \mathrm{MHz}$, but the type 2 phase velocity spectra at 16 and $50 \mathrm{MHz}$ (not shown here) matched exactly.

To summarize, then, the gradient term makes the electrojet region unstable to longitudinal plasma waves most of the time in the electrojet region, but putting reasonable values of the ionospheric parameters into the growth rate expression reveals that the unstable wavelengths will never be short

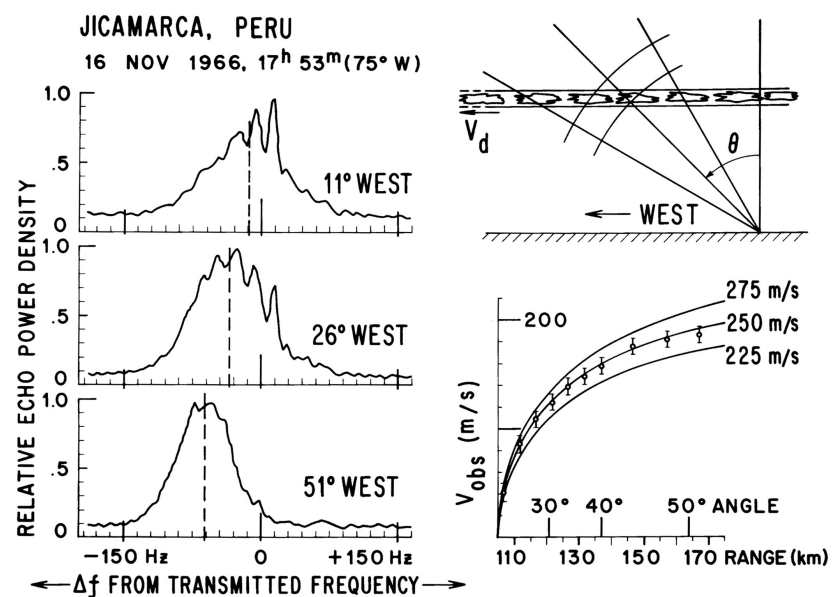

Fig. 9. Simultaneous type 2 echo Doppler spectra at $50 \mathrm{MHz}$ at different zenith angles. The dashed lines show the means, which fit a sine dependence on the zenith angle for a $250 \mathrm{~m} / \mathrm{s}$ velocity (from Balsley, 1969).

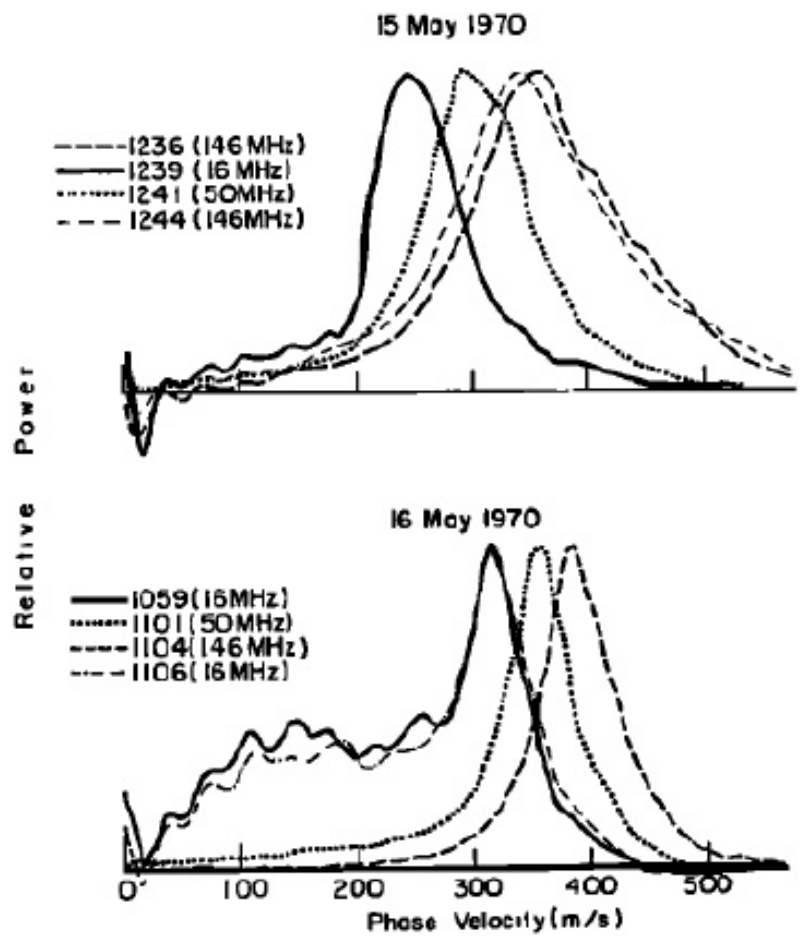

Fig. 10. Comparisons of type 1 spectra (mixed with some type 2 at $16 \mathrm{MHz}$ on $16 \mathrm{May}$ ). The first and last observation of each group were made at the same frequency. Note the systematic shift in phase velocity with radar frequency. Type 2 echoes were not seen at $146 \mathrm{MHz}$ (from Balsley and Farley, 1971).

enough to produce the echoes that VHF radars routinely observe, as long as the inertial term (1) in Eq. (16) is negligible. A more difficult observation to explain is the fact 


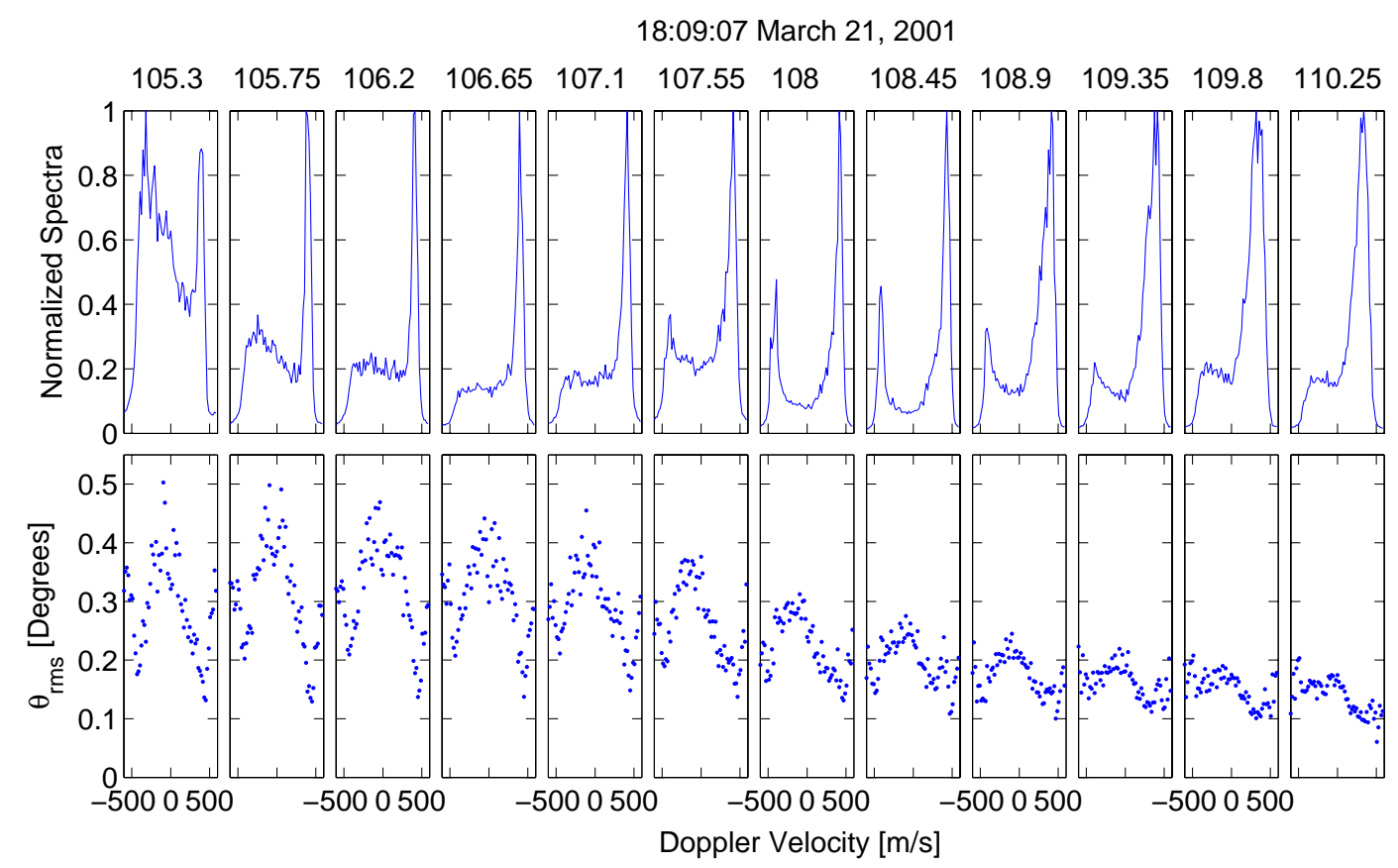

Fig. 11. Type 1 echoes from $105-110 \mathrm{~km}$ above Jicamarca in 2001 . Note the simultaneous presence of both positive and negative peaks at times in the power spectra in the top row. The bottom row shows aspect angle data discussed below (from Lu et al., 2008).

that we see strong radar echoes even with a vertically directed radar. It is important to realize that the Bragg scattering condition is a vector relationship that tells us that radar back-scattering from a sinusoidal plasma wave occurs only if $\mathbf{k}_{\text {plasma wave }}=2 \mathbf{k}_{\text {radar }}$. For the large Jicamarca $50 \mathrm{MHz}$ radar this means that 3-m wavelength plasma waves propagating vertically are somehow excited (often strongly) by the horizontal electron flow. How can this happen when $\mathbf{k} \cdot \mathbf{V}_{d}$ is apparently zero? In other words, how does the horizontal electron flow of the electrojet current couple energy into vertically propagating plasma waves with short wavelengths? These and related questions have led to a series of assumptions that have been made explicitly or tacitly in the equatorial literature for many years.

\subsection{Common assumptions for the equatorial geometry}

1. First of all, there is overwhelming radar and rocket evidence from Jicamarca and elsewhere that the "local background" electric field is not just the dynamo field plus the larger vertical polarization field that arises to maintain a divergence free current flow even though the vertical conductivity above and below the electrojet is nearly zero. The (almost) ever present large scale wave fields strongly affect the background (the drift velocity term in Eq. 15), and so one cannot (as is sometimes implicitly done) automatically assume that there is a substantial "flow angle" between the directions of $\mathbf{k}_{\text {radar }}$ and $\mathbf{V}_{d}$, especially for the type 1 radar echoes. As an extreme example of this flow angle problem, consider the fact that, using the vertically pointing Jicamarca radar, we often observe (see Fig. 11 for example) daytime type 1 echoes with both positive and negative Doppler shifts at the same time and the same altitude!

This apparent paradox is explained by interferometry and imaging data (e.g., Farley et al., 1981; Hysell et al., 2007, and several earlier references therein) that show that the upand down-traveling waves come from different portions of the (fairly small, usually) scattering volume, when the length of the dominant large-scale waves is smaller than the zonal dimension of the scattering volume (as it is during the day, but not at night). In other words, roughly horizontal electric fields associated with the large-scale (kilometers) waves are as strong as or perhaps even somewhat stronger than the mean vertical electric fields, and so there will be large "local" background drift velocities more or less parallel to the radar $k$-vector for a wide range of zenith angles (including zero). Figure 12 shows a cartoon of the basic idea. Within a typical radar scattering volume there can be a wide assortment of local drift velocities $\mathbf{V}_{d}$.

This hypothesis of turbulent electric fields has been well verified by rocket observation made by Pfaff et al. (1987a) and shown in Fig. 6. As Kelley et al. (2008) point out, these data, plus the Brazilian observations of Pfaff et al. (1997), provide in-situ verification of the sketch in Fig. 12, which was based only on radar data available at that time, for the daytime electrojet with a positive density gradient. 
These (large-scale) wave electric fields are strong enough to directly excite the vertically traveling meter-scale waves that VHF radars observe (type 1 echoes). These are sometimes referred to as "two-step" instabilities.

2. Next, since we see radar echoes that we call type 2 at Jicamarca at all zenith angles (including zero for vertical radar pointing), even when the dynamo electric field is weak and the growth rate predicted by Eq. (16) is negative for $3 \mathrm{~m}$ waves (but positive for longer wave lengths), we are forced to assume that the short waves result from a turbulent cascade from long wavelengths to short. This cascade leads to an observed dependence of the measured mean Doppler shift on the sine of the zenith angle, as we saw in Fig. 9. The drift velocity to be used in Eq. (15) is then the mean, not the very local velocities sketched in Fig. 12. This turbulent cascade also affects the aspect angles, as discussed in the next section.

3. When the dynamo field is strong enough, we see type 1 radar echoes with narrow frequency spectra that have Doppler shifts corresponding to velocities comparable to the ion-acoustic velocity (see Fig. 11). Furthermore, and perhaps somewhat surprisingly, to first order this observed Doppler shift does not depend on the radar zenith angle (see Fig. 8), in contrast to the type 2 echo case. (An exception to this behavior is the example of type 1 echoes at Jicamarca during a very strong counter electrojet, described by Woodman and Chau, 2002).

The more or less generally accepted hypotheses for the normal electrojet behavior are that (1) the type 1 echoes are from plasma waves that are directly excited $\left(\gamma_{k}>0\right)$ with a wave vector of $2 \mathbf{k}_{\text {radar }}$ by the highly turbulent drift velocities and electric fields associated with the large scale ( $\sim$ hundreds of meters or longer) waves, (2) the first two terms in Eq. (16) dominate the growth rate, and (3) in the nonlinear limit in which the mean growth rate goes to zero, the unspecified NL processes somehow conspire to force these two terms to cancel each other out. Hence the phase velocity is always approximately $C_{S}$ and not the value given in Eq. (15) if we take $\mathbf{V}_{d}$ to be the more or less horizontal mean. Also, the threshold phase velocity is probably not exactly the isothermal value of $C_{S}$, either because of neglected thermal effects and/or because of nonlinear processes. The question of the effect of thermal processes on $C_{S}$ has been discussed in considerable detail in several recent papers, especially by Kissack et al. (2008a,b).

These thermal processes and the exact values of the type 1 velocity are not the focus of our concern here, however. In fact, we do not yet know if the dominant nonlinear limiting mechanism is two-dimensional (aspect angle effects are unimportant) or three-dimensional (coupling to damped waves propagating at off-perpendicular angles is the primary loss mechanism). There is a considerable literature covering analytical and numerical studies of 2-D mode coupling; see for example St.-Maurice and Hamza (2001), Otani and Oppenheim (2006), Oppenheim et al. (2008a), and earlier references therein. Unfortunately numerical simulations are still

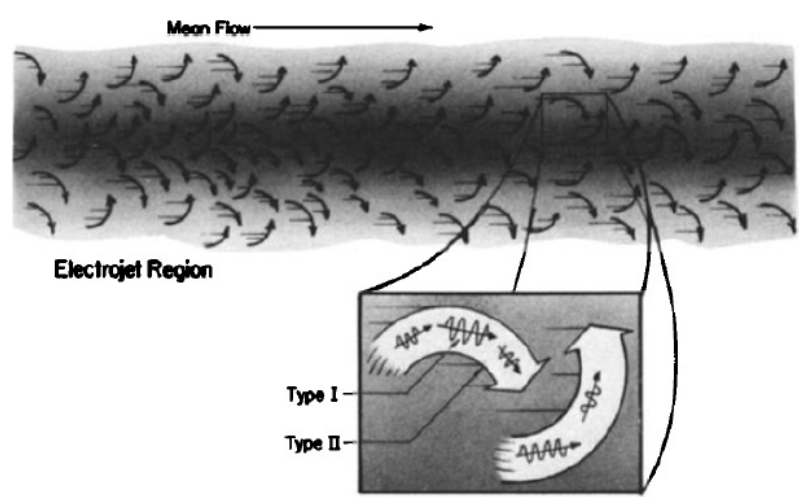

Fig. 12. A sketch of the irregular electron velocity field in the electrojet. Large scale instabilities produce $\boldsymbol{E} \times \boldsymbol{B}$ velocity perturbations comparable to the mean values. The "local" velocities then drive small scale ion-acoustic instabilities, even in the vertical direction (from Farley and Balsley, 1973).

not yet able to deal well with both the coupling to small aspect angles and the 2-D processes simultaneously with sufficient resolution.

In the high latitude (auroral) E region much of the physics is the same, but there are some major differences: (1) the driving electric fields are often much stronger at high latitudes, (2) the magnetic field is nearly vertical, so the important density gradients for the instability are horizontal, and (3) there are gradients in density, electron and ion temperatures, and collision frequencies parallel to the magnetic field that may affect the aspect sensitivity (the size of $\theta_{\text {rms }}$; see Eq. 17). Furthermore, the different radar geometry makes it much harder to measure these aspect angles accurately with interferometry at high latitudes.

\subsection{Magnetic aspect angles}

An important parameter of the unstable electrojet plasma waves is the aspect angle $\theta$ in Eq. (17), which is the angle by which the plasma wave vector deviates from perpendicular to the magnetic field. This parameter is important because even a small component of $\mathbf{k}$ parallel to $\mathbf{B}$ increases wave damping and might cause electron heating, as is observed at high latitudes. Space limitations prevent going into this topic in detail here, but at least a few remarks are in order.

Using radar interferometry techniques with antennas spaced along a magnetic north-south line, it is possible to measure $\theta$, or more particularly $\theta_{\mathrm{rms}}$, the rms deviation of $\theta$ from zero, very accurately at the equator. This was first done at Jicamarca by Kudeki and Farley (1989), who measured angles of a few tenths of a degree that decreased slowly with increasing altitude and also depended on the Doppler shift and the strength of the driving terms. More recently Lu et al. (2008) have revisited and extended these measurements, including early evening echoes above the normal electrojet and 


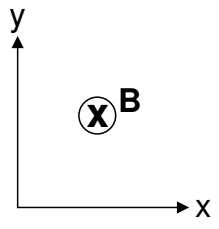

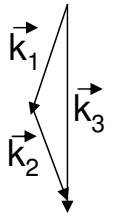

(a)

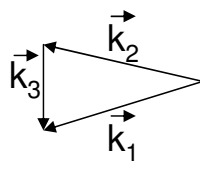

(b)

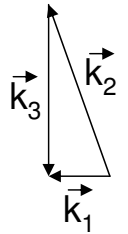

(c)
Fig. 13. Possible 3-wave coupling interactions. In (a) two long unstable waves drive a shorter wave, in (b) two short waves traveling roughly horizontally drive a longer (damped) wave traveling vertically, and in (c) a single long unstable horizontal wave drives two damped shorter waves traveling nearly vertically.

daytime " $150 \mathrm{~km}$ " echoes. Some of the observations can be explained, qualitatively at least, by nonlinear mode coupling arguments of the sort described in the next section. One sample of the recent observations is given in Fig. 10. The bottom panels of that figure show angles as small as $0.1^{\circ}$ corresponding to the type 1 spectral peaks, but with values of the order of $0.4^{\circ}$ for small Doppler shifts at some altitudes.

\subsection{Nonlinear mode coupling}

The data already presented make a strong case for the importance of nonlinear mode coupling. It is easily shown that, no matter what the nonlinear process is, if two waves combine to form a third wave, they must obey the following rule: if waves $\left(\boldsymbol{k}_{\mathbf{1}}, \omega_{1}\right)$ and $\left(\boldsymbol{k}_{\mathbf{2}}, \omega_{2}\right)$ combine to form wave $\left(\boldsymbol{k}_{\mathbf{3}}, \omega_{3}\right)$ then

$\boldsymbol{k}_{\mathbf{3}}=\boldsymbol{k}_{\mathbf{1}}+\boldsymbol{k}_{\mathbf{2}} \quad \omega_{3}=\omega_{1}+\omega_{2}$

Note that the first equation above is a vector addition but the second is not. One or two of the waves must be growing and feeding energy to the remaining wave(s). This process is illustrated in the vector diagrams of Fig. 13.

Cases (a) and (c) in the figure represent a cascade from long wavelengths to short, the sort of process thought to be responsible for type 2 echoes. Case (b) is likely to be responsible for echoes that are sometimes seen by vertically pointing radars in regions where there are no large scale waves (no density gradients) but strong electric fields; see Fig. 5, for example, above $105 \mathrm{~km}$. This mode coupling has implications for the echo aspect angles also, as mentioned above. Coupling energy from long waves to short (e.g., case a) tends to reduce the aspect angle, whereas coupling from short waves to long (e.g., case b) tends to increase it (Kudeki and Farley, 1989; Lu et al., 2008).

\subsection{Kinetic theory calculations}

Several papers (e.g., Ossakow et al., 1975; Schlegel, 1983; St.-Maurice and Schlegel, 1983) have discussed linear kinetic theory for the auroral zone E-region case, especially when the electrojet is very strongly driven, with $\boldsymbol{E} \times \boldsymbol{B}$ drift velocities considerably larger than the ion-acoustic velocity. In this situation the linear growth rate, ignoring all gradients, maximizes for slightly off perpendicular wave vectors. As an example, the calculations of Fig. 5a of St.-Maurice and Schlegel (1983) assumed a drift velocity of $1 \mathrm{~km} / \mathrm{s}$ and a wavelength of $1 \mathrm{~m}$. For ionospheric parameters appropriate to $105 \mathrm{~km}$ in the auroral zone, and with electron-to-ion temperature ratios somewhat larger than unity, they found a mild (double) maximum in the growth rate for angles between $\mathbf{k}$ and $\mathbf{B}$ of $90^{\circ} \pm 0.4^{\circ}$, which would mean a value of $\theta_{\text {rms }}$ of perhaps $0.5^{\circ}$ or so, which is much larger than the values we see for type 1 echoes at the equator. The phase velocity of these primary waves was about $650 \mathrm{~m} / \mathrm{s}$, i.e., less than the drift velocity but more than the ion-acoustic velocity. The same paper made the (very) arbitrary assumption that the wave intensity (proportional to radar echo power) was proportional to the linear growth rate, leading to a Doppler spectrum that is not sharply peaked (their Fig. 1a). The upshot is that the linear kinetic theories so far proposed for the auroral zone predict neither the observed equatorial Doppler power spectra nor the $\theta_{\text {rms }}$ values.

More recently Kissack et al. (2008a) and Kissack et al. (2008b) have written papers discussing in great detail thermal effects and their relation to aspect angles and flow angles. The problem with this is that it is dangerous to assume large flow angles, in particular, since, as we have seen, large scale velocities and electric fields are likely to be quite turbulent, at least at the equator, and radars will always respond most strongly to the regions where the flow angle is small. But large scale waves may not be so important in the auroral zone, since there the density gradients perpendicular to $\mathbf{B}$ that are important are horizontal, not vertical.

\subsection{Numerical simulations and growth saturation}

Computer power continues to increase and simulations have advanced to the point that they are now close to being able to provide us with some answers to key questions such as: What are the dominant nonlinear processes that limit unstable wave growth and thereby determine the wave spectrum that the radars see? Meers Oppenheim and his colleagues have pioneered in the recent work (see, e.g., Oppenheim et al., 2008a, and earlier references therein). These results, especially the movies that you can't put in a paper figure, show how the waves can limit in a purely 2-D simulation in a plane perpendicular to $\mathbf{B}$. The simulations usually ignore zero order electron density gradients and drive the instability strongly, for computational cost reasons. The ion-acoustic waves grow rapidly and develop strong electric fields and gradients of their own, generating new waves traveling more or less perpendicularly to the primary waves. These secondary waves eventually destroy the coherence of the original waves, which break up somewhat chaotically and then begin to regroup and 
start growing again. The predicted (type 1) phase velocities are fairly close to $C_{s}$, taking into account some heating.

So we can certainly limit the wave growth without invoking the third dimension, but coupling to damped waves with a small parallel (to B) component of $\mathbf{k}$ might turn out to be the primary limiting process. As already mentioned, we know from interferometry measurements at Jicamarca (Kudeki and Farley, 1989; Lu et al., 2008) that $k$-vectors can depart from normal to $\mathbf{B}$ by a few tenths of a degree, but how important is this? Analytic "pencil and paper" theories cannot sort out the competition between the 2-D and 3-D processes. Simulations are our main hope of finding out what really matters. Of course trying to do sufficiently detailed (i.e., realistic) simulations in three dimensions requires enormous computing power, but some exciting work has already been done (Oppenheim et al., 2008b).

\section{Some final questions}

This is far from a complete review of the subject, but it should provide some background for people relatively new to the field and perhaps a little food for thought for those of us working actively on electrojet instabilities. Some questions to think about include:

1. How important are flow angle and related thermal effects at the equator? How much do they (or don't they) affect the wave phase velocities and the aspect angles? How relevant to the equatorial case is the work done for the auroral zone? The measured aspect angles $\theta_{\text {rms }}$ at the equator are very small, in contrast (perhaps?) to the auroral zone. In any case, it seems clear that it is a serious mistake to assume that the electron flow is even remotely laminar over a significant range of space and time (e.g., the radar scattering volume and integration time), although this can happen occasionally during a daytime counter electrojet.

2. Is the dominant nonlinear process that limits wave growth primarily 2-D or 3-D? Will Moore's law concerning computer power growth continue to hold long enough to give us the computer power to do really realistic 3-D computer simulations? By "realistic" I mean a simulation from which you can calculate the properties of scattered radar pulses and in-situ fields and show that these agree with actual radar and rocket observations. Once you can really do that, the computer can tell you what the dominant nonlinear processes are. For the equator, at least, the simulations should not be driven too hard, insofar as that is practical.

3. How different are the auroral and equatorial zones? Is the physics essentially the same, except for the fact that the auroral zone electric fields are often much stronger? Or is it important that in the auroral zone there are gradients parallel to the magnetic field in electron density, electron and ion temperatures, and collision frequencies?

4. Interesting satellite and Jicamarca radar data were taken during the huge November 2004 magnetic storm, during which the maximum driving electric fields at Jicamarca were several times their normal values. Comparing the electric and magnetic field data suggests (M. C. Kelley, private communication, 2009) that the Cowling conductivity was substantially less than its normal value during the periods of extreme electric fields, presumably because of energy losses associated with the generation of strong plasma waves. Can simulations perhaps quantitatively explain this also? Is there other confirming evidence? Is this something that global models need to worry about?

Acknowledgements. This work was supported by the Atmospheric Sciences Division of the National Science Foundation through Cooperative Agreement ATM-0432565 with Cornell University. This Agreement also supports the operations of the Jicamarca Radio Observatory. I also thank Mike Kelley and Wesley Swartz for help in preparing this tutorial.

Topical Editor K. Kauristie thanks M. Uspensky and another anonymous referee for their help in evaluating this paper.

\section{References}

Balsley, B. B.: Some characteristics of non-two-stream irregularities in the equatorial electrojet, J. Geophys. Res., 74, 2333-2347, 1969.

Balsley, B. B. and Farley, D. T.: Radar studies of the equatorial electrojet at three frequencies, J. Geophys. Res., 76, 8341-8351, 1971.

Cohen, R. and Bowles, K. L.: Secondary irregularities in the equatorial electrojet, J. Geophys. Res., 72, 885-894, 1967.

Farley, D. T. and Balsley, B. B.: Instabilities in the equatorial electrojet, J. Geophys. Res., 78, 227-239, 1973.

Farley, D. T., Ierkic, H. M., and Fejer, B. G.: Radar interferometry: A new technique for studying plasma turbulence in the ionosphere, J. Geophys. Res., 86, 1467-1472, 1981.

Fejer, B. G., Farley, D. T., Balsley, B. B., and Woodman, R. F.: Vertical structure of the VHF backscattering region in the equatorial electrojet and the gradient drift instability, J. Geophys. Res., 80, 1313-1324, 1975.

Forbes, J. M. and Lindzen, R. S.: Atmospheric solar tides and their electrodynamic effects. II. The equatorial electrojet, J. Atmos. Terr. Phys., 38, 911-920, 1976.

Hysell, D. L., Drexler, J., Shume, E. B., Chau, J. L., Scipion, D. E., Vlasov, M., Cuevas, R., and Heinselman, C.: Combined radar observations of equatorial electrojet irregularities at Jicamarca, Ann. Geophys., 25, 457-473, 2007, http://www.ann-geophys.net/25/457/2007/.

Kelley, M. C.: The Earth's Ionosphere, Academic Press, San Diego, California, 1989.

Kelley, M. C., Cuevas, R. A., and Hysell, D. L.: Radar scatter from equatorial electrojet waves: An explanation for the constancy of 
the type 1 Doppler shift with zenith angle, Geophys. Res. Lett., 35, L04106, doi:10.1029/2007GL032848, 2008.

Kissack, R. S., Kagan, L. M., and St.-Maurice, J.-P.: Thermal effects on Farley-Buneman waves at nonzero aspect and flow angles. I Dispersion relation, Phys. Plasma, 15, 022901, doi:10.1063/1.2834275, 2008a.

Kissack, R. S., Kagan, L. M., and St.-Maurice, J.-P.: Thermal effects on Farley-Buneman waves at nonzero aspect and flow angles. II Behavior near threshold, Phys. Plasma, 15, 022902, doi:10.1063/1.2834276, 2008b.

Kudeki, E. and Farley, D. T.: Aspect Sensitivity of equatorial electrojet irregularities and theoretical implications, J. Geophys. Res., 94, 426-434, 1989.

Lu, F., Farley, D. T., and Swartz, W. E.: Spread in aspect angles of equatorial $E$ region irregularities, J. Geophys. Res., 113, A11309, doi:10.1029/2008JA013018, 2008.

Oppenheim, M. M., Dimant, Y., and Dyrud, L. P.: Large-scale simulations of 2-D fully kinetic Farley-Buneman turbulence, Ann. Geophys., 26, 543-553, 2008a, http://www.ann-geophys.net/26/543/2008/.

Oppenheim, M. M., Dimant, Y., Tambouret, Y., and Dyrud, L. P.: Large-scale simulations of Farley-Buneman turbulence in 2D and $3 \mathrm{D}$ and hybrid gradient drift simulations, conference presentation, 2008b.

Ossakow, S. L., Papadopoulos, K., Orens, K., and Coffey, T.: Parallel propagation effects on the type 1 electrojet instability, J. Geophys. Res., 80, 141-148, 1975.

Otani, N. F. and Oppenheim, M. M.: Saturation of the FarleyBuneman instability via three-mode coupling, J. Geophys. Res., 111, A03022, doi:10.1029/2005JA011215, 2006.

Pfaff, R. F., Kelley, M. C., Fejer, B. G., Maynard, N. C., and Baker, K. D.: In-situ measurements of wave electric fields in the equatorial electrojet, Geophys. Res. Lett., 9, 688-691, 1982.
Pfaff, R. F., Kelley, M. C., Kudeki, E., Fejer, B. G., and Baker, K. D.: Electric field and plasma density measurements in the strongly driven daytime equatorial electrojet, 1, The unstable layer and gradient drift waves, J. Geophys. Res., 92, 1357813596, 1987a.

Pfaff, R. F., Kelley, M. C., Kudeki, E., Fejer, B. G., and Baker, K. D.: Electric field and plasma density measurements in the strongly driven daytime equatorial electrojet, 2, Two-stream waves, J. Geophys. Res., 92, 13597-13612, 1987 b.

Pfaff, R. F., Acuna, M. H., Marionni, P. A., and Trevedi, N. B.: DC polarization electric field, current density, and plasma density measurements in the daytime equatorial electrojet, Geophys. Res. Lett., 24(13), 1667-1670, 1997.

Prakash, S., Subbaraya, B. H., and Gupta, S. P.: Rocket measurements of ionization irregularities in the equatorial ionosphere at Tumba and identification of plasma irregularities, Indian J. Radio Space Phys., 1, 72-80, 1972.

Rishbeth, H. and Garriott, O. K.: Introduction to Ionospheric Physics, Academic Press, New York, 1969.

Schlegel, K.: Interpretation of auroral radar experiments using a kinetic theory of the two-stream instability, Radio Sci., 18, 108$118,1983$.

St.-Maurice, J.-P. and Hamza, A. M.: A new nonlinear approach to the theory of $E$ region irregularities, J. Geophys. Res., 106, 1751-1760, 2001.

St.-Maurice, J.-P. and Schlegel, K.: A theory of coherent radar spectra in the auroral $E$ region, J. Geophys. Res., 88, 4087-4095, 1983.

Woodman, R. F. and Chau, J. L.: First Jicamarca radar observations of two-stream $E$ region irregularities under daytime counter equatorial electrojet conditions, J. Geophys. Res., 107(A12), 1482, doi:10.1029/2002JA009362, 2002. 